\title{
Design and evaluation of a subcutaneous contraceptive implant training simulator
}

\author{
Samuel K.K. Dery $^{1, *}$ | Elsie E. Kaufmann ${ }^{2}$ | David Marzano ${ }^{3}$ | Michael Deininger $^{4}$ | \\ Charity K. Asem ${ }^{5}$ | Kathleen H. Sienko ${ }^{6}$
}

${ }^{1}$ Department of Biostatistics, School of Public Health, University of Ghana, Accra, Ghana

${ }^{2}$ Department of Biomedical Engineering, University of Ghana, Accra, Ghana

${ }^{3}$ Department of Obstetrics and Gynecology, University of Michigan, Ann Arbor, MI, USA

${ }^{4}$ Design Science Program, University of Michigan, Ann Arbor, MI, USA

${ }^{5}$ School of Public Health, University of Ghana, Accra, Ghana

${ }^{6}$ Department of Mechanical Engineering, University of Michigan, Ann Arbor, MI, USA

\section{${ }^{*}$ Correspondence}

Samuel K.K. Dery, Department of Biostatistics, School of Public Health, University of Ghana, Legon, Accra. Emails: skdery@ug.edu.gh; skdery@yahoo.com

\section{Funding Information}

Ghana-Michigan Post-doctoral And Research Trainee NEtwoRk - Investing in Innovation (PARTNER II)

\begin{abstract}
Objective: To design and fabricate a subcutaneous contraceptive implant insertion simulator, and to characterize the performance of nursing students trained with and without the simulator.

Method: A cross-sectional study was conducted on nursing students in Ghana who had no previous training in the insertion of contraceptive implants. They were given standardized training in insertion of implants from 25 April to 26 April, 2016, and then were randomly assigned to an intervention or control group. The control group watched insertions of live implants while the intervention group practiced using the simulator. Local materials were used to fabricate the simulator. The performance of both groups was assessed after the training.

Results: The participants consisted of 50 nursing students. Those in the intervention group were more likely to: insert the implant accurately ( $95.2 \%$ vs $78.4 \%, P<0.001$ ); take less time to complete an insertion (mean of 33.6 seconds vs 42.2 seconds, $P<0.001$ ); and commit fewer errors (1.9 vs $2.5, P=0.005$ ) compared to the control group. In addition, participants rated the simulator high on $11 / 11$ of the product requirements with the teaching (93.2\%), learning (91.4\%), and skill acquisition (88.6\%) requirements being the highest rated.

Conclusion: A low-cost, locally fabricated simulator is an effective tool for augmenting the current training protocol by improving insertion skills of contraceptive implants.

\section{KEYWORDS}

Community health nurses; Contraceptive implant; Family planning; Ghana; Implanon; Nexplanon; Novice; Simulation
\end{abstract}

\section{1 | INTRODUCTION}

In Ghana, there is an unmet demand for family-planning services. In the 2014 Ghana Demographic and Health Survey, $29.9 \%$ of married women had unmet needs for family-planning services. In addition, only $26.7 \%$ of currently married women used contraception: $22.2 \%$ used modern methods, with the three most popular modern methods being injectables (8.0\%), implants (5.2\%), and pill (4.7\%). ${ }^{1}$ Contraceptive implants are a very effective, long-acting, reversible family-planning method with the potential to contribute to the family-planning needs of most women. ${ }^{2}$ They prevent pregnancy for up to 3-5 years after administration with no regular action by the user and no routine clinical follow-up required. ${ }^{3,4}$ Implants are reported to have high rates of continuation and client satisfaction among users in recent years. ${ }^{5,6}$ Contraceptive implants offer promising opportunities for addressing the high and growing unmet need for modern contraceptives in sub-Saharan Africa. ${ }^{5}$ Currently, three main types of implants (Jadelle, Sino-implant, and Implanon/Nexplanon) are being used in Ghana. Jadelle and Sino-implant are composed of 
two thin, flexible rods, each containing $75 \mathrm{mg}$ levonorgestrel and are currently labeled for five years of use. Implanon, and its latest version Nexplanon, is a single-rod, hormonal implant that contains etonogestrel and is labeled for three years of use. ${ }^{7}$

A key factor contributing to the low usage of modern methods, particularly implants, is a shortage of trained staff, particularly those skilled in providing contraceptive insertion services. ${ }^{8-10}$ Insertion of implants requires skilled staff; if not properly inserted, implants can cause pain, vary in their effectiveness, and lead to difficult removal frequently requiring surgical interventions with the possibility of additional complications. ${ }^{11} \mathrm{~A}$ recent $\mathrm{WHO}$ review of the evidence on the safety of the insertion and removal of implants concluded that auxiliary nurses could deliver implant services with targeted monitoring and evaluation. ${ }^{12}$ Some studies have also shown that community health nurses (CHNs) can indeed administer contraceptive implants, suggesting that if more $\mathrm{CHNs}$ are trained to provide contraceptive implant services, coupled with the creation of demand, the use of implants may increase. ${ }^{8,13}$

However, training $\mathrm{CHNs}$ remains a major challenge in Ghana as the current mode of training is primarily conducted through lectures, videos, and observations of clinical experts performing insertion of implants. This approach lacks the opportunity for trainees to gain proficiency through practice. We hypothesized that a simulatorbased training curriculum, when compared to video-based exposure to methods of insertion, would improve insertion skills in novices and improve confidence of providers.

The aim of the present study was to develop and assess a subcutaneous contraceptive implant insertion simulator for training healthcare workers to appropriately insert contraceptive implants. The results of this research are expected to contribute to increased access to longterm contraceptive products/services for women through improved training of healthcare workers.

\section{2 | MATERIALS AND METHODS}

The project was a cross-sectional study and was divided into three phases: design; development; and evaluation of the simulator.

In the design phase, physicians, nurses, midwives, and engineers from the University of Michigan and University of Ghana were consulted to develop the product requirements and engineering specifications for the simulator between December 2014 and January 2015. Healthcare personnel provided critical information, including a detailed understanding of the procedural steps required for performing proper insertion of contraceptive implants, and identified the key anatomical features required to properly simulate the procedure. The product requirements (Table 1 ) were defined and prioritized based on the input from these professionals as well as from the literature. ${ }^{14}$ After determining the requirements, functional decomposition was performed and design concepts were generated. ${ }^{15}$

The concepts were assessed based on their ability to satisfy the product requirements, and three distinct concepts were selected for further assessment. Ethical approval (exempt status) was obtained from the University of Michigan Institutional Review Board (IRB) in
TABLE 1 Product requirements.

\begin{tabular}{|ll}
\hline Priority & Product requirements \\
\hline 1 & Be anatomically correct \\
\hline 2 & Allow user to gain proficiency of insertion process \\
\hline 3 & Provide user and trainer with knowledge of results \\
\hline 5 & Be safe \\
\hline 6 & Be producible in resource-limited setting \\
\hline 7 & Be easy to use by both trainer and trainee \\
\hline 9 & Be low cost \\
\hline 10 & Be reusable \\
\hline 11 & Allow simulation of insertion of major implants used \\
\hline
\end{tabular}

February 2015 to solicit feedback from clinical experts to inform iterations to the design of the final simulator.

Forty-one clinical experts from the Korle Bu Teaching Hospital in Accra, Ghana assessed mock-ups of the three selected concepts for their suitability in August 2015. Two of the concepts (17 and 16 clinicians ranked them the highest, respectively) were preferred over the third concept. Based on the results of the initial assessment, the prototype with the highest score (subsequently referred to as the simulator) was fabricated (Fig. 1) using materials that were locally produced in Ghana (PVC pipe, latex foam, cotton, and leather) and was used for formal evaluation to enable the trainee to learn the entire process of implant insertion. The process is briefly described as follows: the "patient" is asked to lie on her back with her arm resting on an arm support; the implant insertion package is opened; the insertion site is cleaned with antiseptic; the optimal

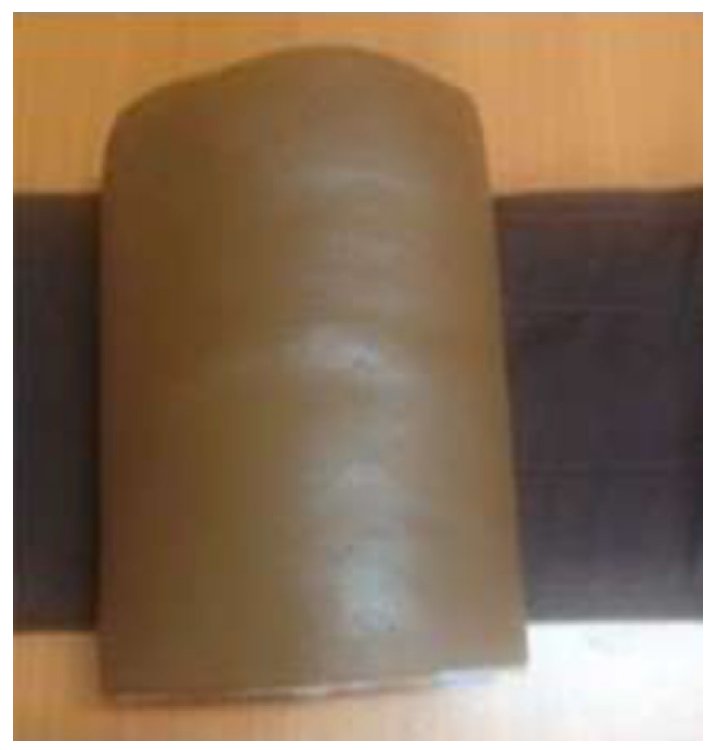

FIGURE 1 Sample of the simulator. 


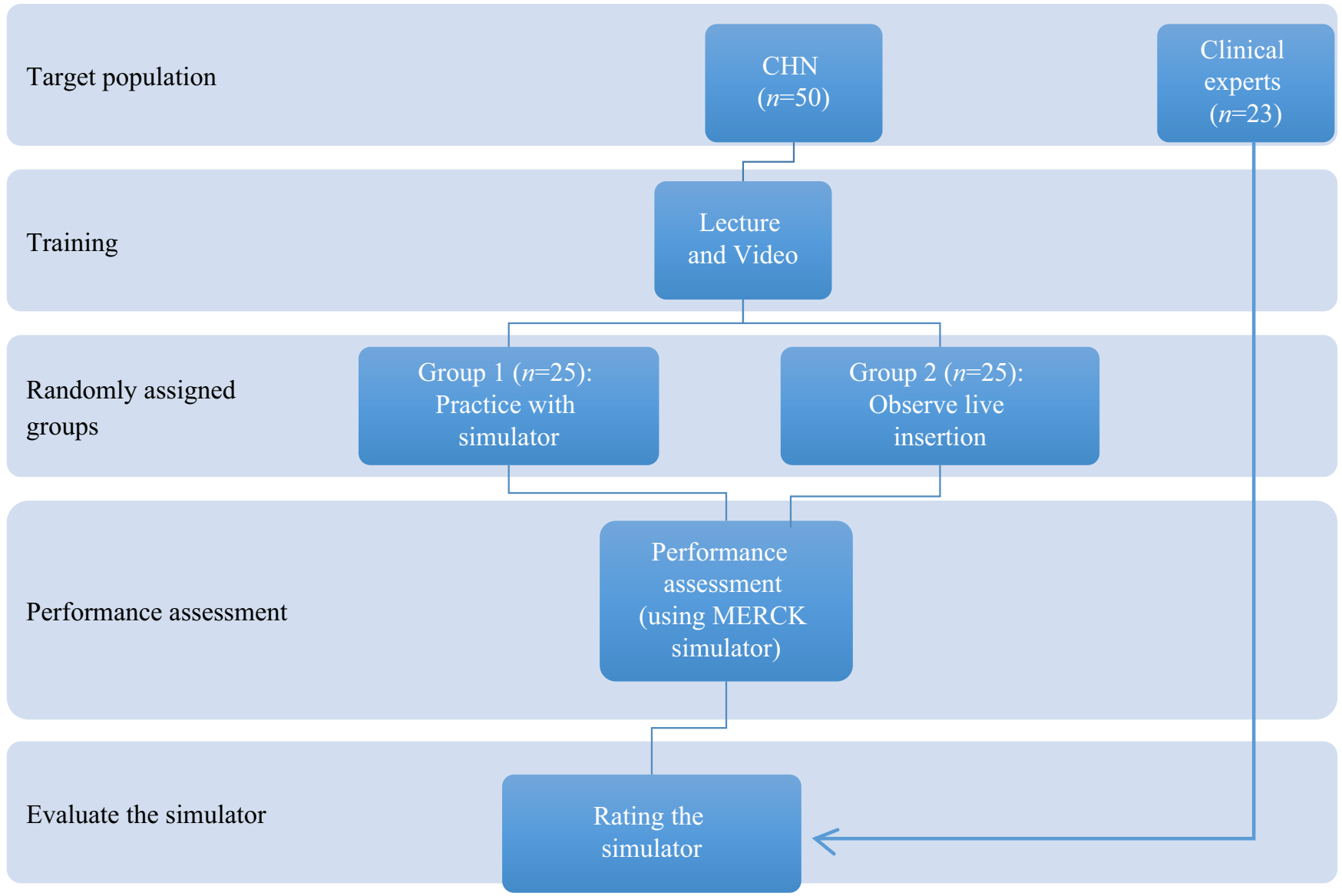

FIGURE 2 Study design.

insertion site is determined; local anesthetics are applied; the implant is then inserted.

The simulator included a semi-hollow, cylindrical, PVC base to mimic bone and a piece of cloth for firmly securing it to the arm of the patient (person to receive the implant) during testing or training, to enable the trainee to more accurately replicate typical interactions with the patient and practice the steps outlined above.

Figure 2 provides an illustration of the study design used to evaluate the simulator. Fifty community health nursing students in their second year of training, and with no previous training in the insertion of contraceptive implants, were selected from the $\mathrm{CHNs}$ training school in Winneba, in the Central Region of Ghana. The sample size was calculated based on the ability to detect a difference of $30.0 \%$ in the accuracy of insertion between the control and intervention groups at a power of $80.0 \%$. The study was approved by the Ghana Health Service Ethics Review Committee and participants gave written informed consent before the training. Participants were trained in the processes and steps of Implanon insertion using the current method of training, including lectures and standardized training videos. After the training, participants were randomly assigned to an intervention or control group equally. Participants were assigned serial numbers from 1 to 50 and the Microsoft Excel RANDBETWEEN function was used to generate 25 random numbers between 1 and 50 to form the intervention group, with the balance of the participants forming the control group.
The control group observed actual insertions of implants in the clinical setting (six insertions) performed by midwives (standard method currently in use) while the intervention group practiced insertions of Implanon using the simulator (five insertions each), without watching the midwives perform actual insertions. The accuracy and duration of each insertion were measured. The accuracy of the insertion was assessed by two midwives via visual inspection using the scale in Table 2. Insertions underneath the skin or within the fat layer (code 2 or 3 ) were considered accurate. Time to complete the insertion was measured in seconds from start to finish using a stopwatch. The performance of both groups was then assessed after the training using the MERCK model of Implanon insertion training. ${ }^{16}$ The accuracy of insertion, duration of insertion, and number of errors committed were assessed for each insertion.

TABLE 2 Accuracy of insertion scale.

\begin{tabular}{ll}
\hline Code & Type of insertion \\
\hline 0 & False/no insertion \\
1 & Insertion within the skin \\
\hline 2 & Insertion underneath the skin and partially in the fat layer \\
3 & Insertion in the fat layer \\
\hline 4 & Insertion in the muscle layer \\
\hline
\end{tabular}


TABLE 3 Performance assessment scores.

\begin{tabular}{lcccc}
\hline & $\begin{array}{l}\text { Intervention } \\
\text { group }\end{array}$ & $\begin{array}{l}\text { Control } \\
\text { group }\end{array}$ & All groups & P value \\
\hline $\begin{array}{l}\text { Accuracy of } \\
\text { insertion, } \mathrm{n} \\
(\%)\end{array}$ & $119(95.2)$ & $98(78.4)$ & $217(86.8)$ & $<0.001$ \\
$\begin{array}{l}\text { Insertion } \\
\text { time, } \bar{x}(\mathrm{SD})\end{array}$ & $33.62(1.20)$ & $42.24(1.99)$ & $37.93(1.19)$ & $<0.001$ \\
\hline $\begin{array}{l}\text { Number of } \\
\text { errors } \\
\text { committed, }\end{array}$ & $1.93(0.11)$ & $2.48(0.16)$ & $2.20(0.10)$ & 0.005 \\
$\overline{\mathrm{x}}(\mathrm{SD})$ & & & & \\
\hline
\end{tabular}

After the training and the performance assessment were completed, all participants and 23 clinical experts with knowledge and experience of inserting contraceptive implants tested and evaluated the simulator on 11 key requirements: (1) anatomic fidelity; (2) procedural fidelity; (3) ability to allow the user to gain proficiency; (4) provision of feedback to the user; (5) ease of use (setup); (6) ability to facilitate acquisition of insertion skills; (7) usefulness in teaching implant insertion; (8) usefulness in learning implant insertion; (9) enhancement of confidence of the trainee; (10) provision of realistic practice opportunities; and (11) ease of insertion of implants. They scored each requirement using a Likert scale ranging from 1 (strongly disagree) to 5 (strongly agree). In addition, they provided comments about the simulator and responses to several open-ended questions focused on first impressions, specific training problems the simulator would address, comparison with other methods of training to insert implants, and the main advantages of the simulator. Thematic coding was used to analyze the open-ended questions (Table 5). Stata MP Version 13 was used to analyze the quantitative data. $\chi^{2}$ analysis was used to test the association between the groups and the ratings of the various requirements of the simulator, while a t-test was used to test the performance between the intervention and control groups at a significance level of $95 \%$.

\section{3 | RESULTS}

Table 3 shows the performance of the participants after the assessment. Overall, the number of accurate insertions by all participants was $217(86.8 \%)$, while the mean time taken to complete an insertion was 37.9 seconds and the mean number of errors committed was 2.20. There were significant associations between the groups and the accuracy, duration of insertion, and number of errors committed. The participants in the intervention group were more likely to: insert the implant accurately (119 [95.2\%] of all the insertions by the intervention group were accurate compared to 98 [78.4\%] for the control group, $P<0.001$ ); take less time to complete an insertion (mean time of 33.6 seconds for intervention group compared to 42.2 seconds for control group, $P<0.001$ ); and commit fewer errors (mean number of errors committed was 1.9 compared to 2.5 for the control group, $P=0.005$ ) (Table 3).

Fifty-four (75.0\%) of the participants $(20$ [80.0\%] in the intervention group, 17 [68.0\%] in the control group, and 17 [77.0\%] health professionals) agreed that the simulator was anatomically realistic. Additionally, more than 57 (80.0\%) participants agreed that the simulator was useful for teaching, learning, and acquisition of skills (Fig. 3).

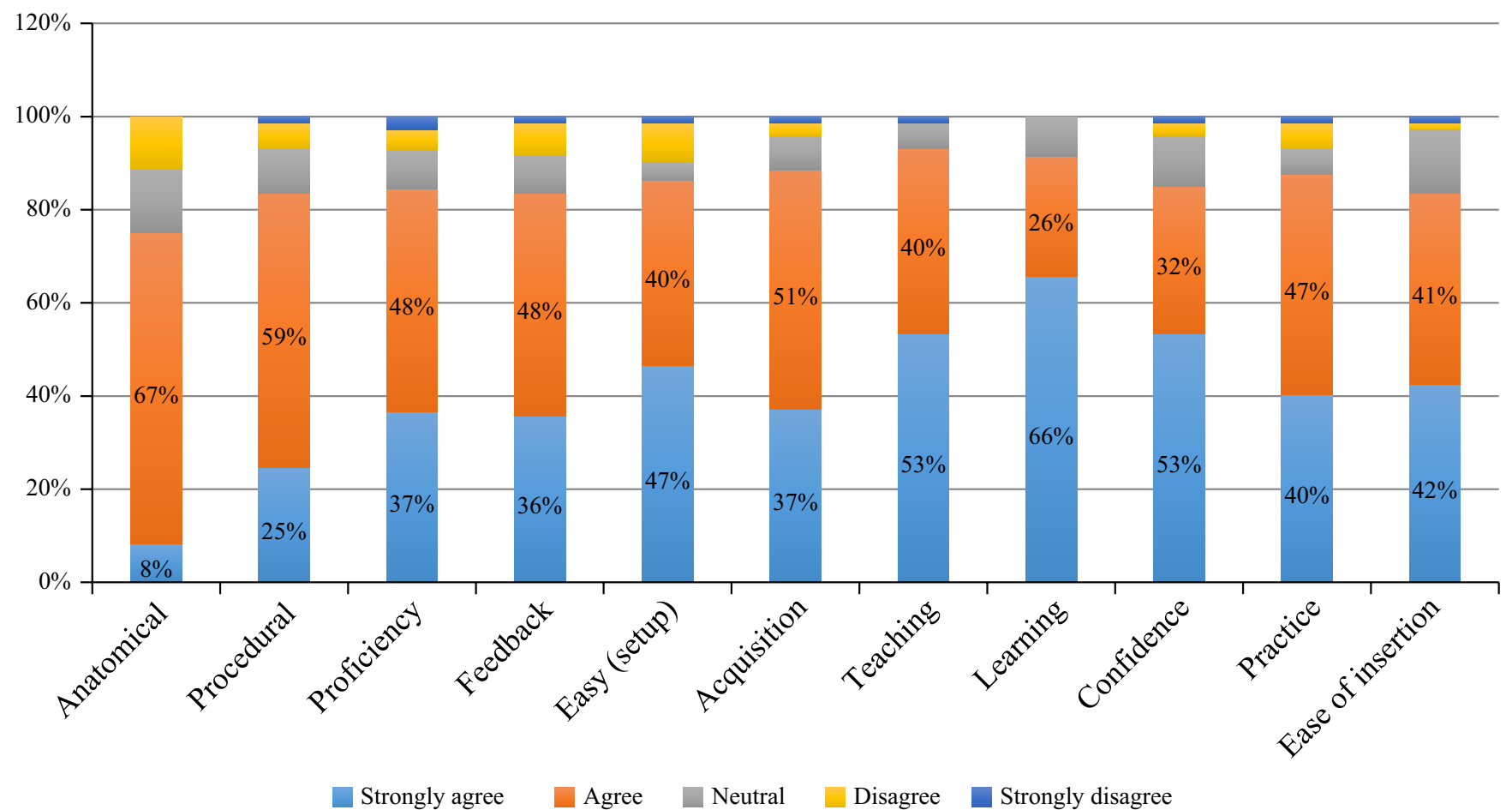

FIGURE 3 Overall rating of the simulator. 
TABLE 4 Simulator ratings.

\begin{tabular}{|c|c|c|c|c|c|}
\hline & $\begin{array}{l}\text { Intervention group } \\
(\mathrm{n}=25)\end{array}$ & $\begin{array}{l}\text { Control group } \\
(n=25)\end{array}$ & $\begin{array}{l}\text { Health professionals } \\
(n=23)\end{array}$ & All participants & \\
\hline Requirement & $\overline{\mathbf{x}}(\mathrm{SD})$ & $\overline{\mathrm{x}}(\mathrm{SD})$ & $\overline{\mathbf{x}}(\mathrm{SD})$ & $\overline{\mathbf{x}}(\mathrm{SD})$ & $P$ value \\
\hline Anatomical fidelity & $3.88(0.13)$ & $3.56(0.16)$ & $3.73(0.18)$ & $3.72(0.09)$ & 0.348 \\
\hline Procedural fidelity & $3.88(0.19)$ & $4.08(0.17)$ & $4.08(0.13)$ & $4.00(0.10)$ & 0.673 \\
\hline Allow user to gain proficiency & $4.12(0.17)$ & $4.24(0.20)$ & $3.95(0.21)$ & $4.11(0.11)$ & 0.588 \\
\hline Ease of use (setup) & $4.08(0.22)$ & $4.32(0.19)$ & $4.26(0.18)$ & $4.22(0.11)$ & 0.662 \\
\hline $\begin{array}{l}\text { Facilitate acquisition of } \\
\text { insertion skills }\end{array}$ & $4.12(0.19)$ & $4.38(0.12)$ & $4.10(0.19)$ & $4.20(0.10)$ & 0.430 \\
\hline $\begin{array}{l}\text { Useful in teaching insertion of } \\
\text { implants }\end{array}$ & $4.36(0.19)$ & $4.48(0.12)$ & $4.47(0.12)$ & $4.44(0.08)$ & 0.806 \\
\hline $\begin{array}{l}\text { Provide realistic practice } \\
\text { opportunities }\end{array}$ & $4.04(0.21)$ & $4.24(0.17)$ & $4.30(0.16)$ & $4.19(0.10)$ & 0.571 \\
\hline Easy to insert implant & $4.00(0.19)$ & $4.16(0.15)$ & $4.52(0.15)$ & $4.22(0.10)$ & 0.088 \\
\hline Average rating of simulator & $4.14(0.13)$ & $4.26(0.08)$ & $4.22(0.13)$ & $4.20(0.56)$ & \\
\hline
\end{tabular}

asignificant.

Overall, the average rating of the simulator for the intervention group was 4.1 (out of a maximum of 5) compared to 4.3 for the control group and 4.2 for the health professionals. There were no significant differences in the ratings of the requirements of the simulator by participants with the exception of the requirement that the simulator should provide feedback to the user (mean rating of 4.3 for intervention and control groups, and 3.7 for health professionals, $P=0.038$ ) as shown in Table 4.

TABLE 5 Themes generated.

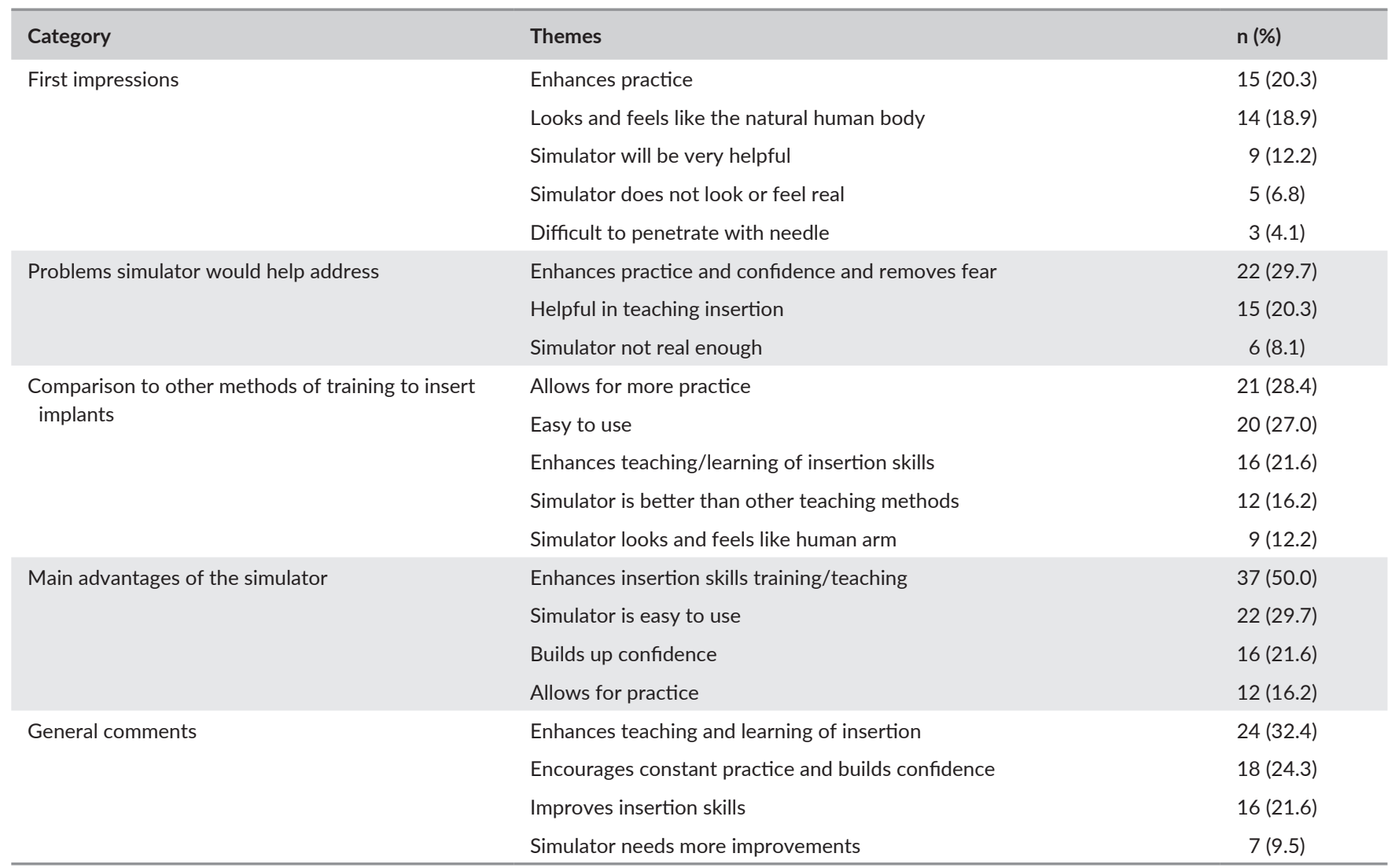


Regarding their first impressions about the simulator, several of the participants commented on its potential for enhancing practice and the realistic look and feel of the simulator (Table 5). The most prevalent themes raised included "looks and feels like human arm" and "enhances the practice of insertion." The following comment from a participant illustrates this finding: "It is designed just like the human arm so it makes practice easy" (a nurse). There were a few critiques of the simulator as well; for example, a student said, "My first impression of the simulator was that it is too soft and enhances insertion errors."

Most participants said the simulator would address the challenge of not having adequate practice before inserting implants in humans and would also address the anxiety that learners may face while performing real insertions. Comments from respondents that illustrate this finding include: "the simulator would prevent any insertion injury, since one has been practicing with the simulator" (a student); and "help build the confidence of the health practitioner who do this insertion and prevent error in inserting the implant on a real human skin" (a student).

When asked how this simulator compares to other methods of training to insert implants, most participants said that the simulator was very useful and provided a practical opportunity to trainees. They described the simulator-based learning as very useful and easy to use (e.g. "Practicing with the simulator is more helpful than you watching a video or watching someone doing it for you to see" [a student]).

On the main advantages provided by this simulator, most respondents said it improves insertion skills, makes practice of insertion easier, and enhances confidence. A student respondent stated, "It gives you the chance to do the inserting even without a human being. It is more or less like a human body so if you are able to do correct insertion on it, then you can do likewise on the human being".

Participants recommended that the simulator should be used in the training schools: "It should be made and used in the training schools to enable students to practice and know how to insert implants ... It should be done such that every student can own one" (a nurse).

\section{4 | DISCUSSION}

This study sought to design, develop, and assess a low-cost, high-fidelity simulator made from local materials for the training of nursing students, and other health professionals, on the insertion of contraceptive implants. The results show that the participants trained with the simulator were more likely to insert the implant accurately, take less time to complete an insertion, and commit fewer errors compared to those trained without the simulator. In addition, participants rated the simulator high on all of the product requirements.

The results of the assessment of the participants' performance show that the intervention group performed better in all areas (accuracy of insertion, duration of insertion, and number of errors committed), suggesting that the simulator would be a useful tool for training nursing students and other healthcare workers in performing insertion of implants. Similar results from simulators developed for learning to manage postpartum hemorrhage, ${ }^{14}$ surgical training, ${ }^{17}$ and breast examination ${ }^{18}$ have also shown positive effects. Simulation-based learning, a method where an environment similar to the real context is created for the trainee to learn the skills required, enables learners to practice extensively and gain experience in a safe, non-threatening environment and has been shown to yield positive results. ${ }^{19,20}$

The high rating of the simulator by health professionals and students also provides evidence to support the use of this simulator for training healthcare workers on the insertion of contraceptive implants. The findings in this study mirror similar ratings assigned to other low-cost simulators developed with local materials. ${ }^{14}$ Consistent with results from similar work carried out in Ghana, ${ }^{14}$ the healthcare professionals with experience in the insertion of implants and the nursing students found the simulator to be realistic and particularly useful for teaching, learning, and the acquisition of skills. In contrast, the current mode of training does not provide students with the opportunity to practice before they undertake insertion of implants in the field. Tools for practicing such insertions are not readily available, particularly for nursing students in training; thus, they only learn through lectures and training videos, and from observing clinical experts when they are working at health facilities. Opportunities for students to practice before performing the insertion of implants on humans are mostly non-existent. This simulator therefore provides the opportunity for students to gain enough practice not only in the act of insertion but also the entire process of inserting implants. It may also boost the confidence of trainees and reduce any anxiety that they may have in the process of inserting implants. This simulator adds to the growing body of evidence that simulator-based training has the potential to help healthcare workers acquire the skills needed to perform various activities. ${ }^{11,19,21,22}$

One limitation of this study is that the intervention group was not asked to also observe actual insertions of implants in the clinical setting (a component of the current standard training procedure) before practicing with the simulator. It is possible that the inclusion of that additional training component may have further improved the performance of the intervention group.

\section{AUTHOR CONTRIBUTIONS}

SKKD developed the concept for the study and was responsible for the study design, analysis and writing of the manuscript. EEK was responsible for the study design and revision of the manuscript. DM was responsible for the study design and revision of the manuscript. MD was responsible for the study design and revision of the manuscript. CKA was responsible for the literature review and revision of the manuscript. KHS developed the concept for the study and was responsible for the study design and revision of the manuscript. All authors approved the final version of the manuscript.

\section{ACKNOWLEDGMENTS}

This project was funded by the Ghana-Michigan Post-doctoral And Research Trainee NEtwoRk - Investing in Innovation (PARTNER II). 
Grant number 1D43TW009353. We also acknowledge the support of the PARTNER II 2014-2015 cohort: Cheryl Moyer, Samuel Oppong, Ashura Bakari, and April Bell.

\section{CONFLICTS OF INTEREST}

The authors have no conflicts of interest.

\section{REFERENCES}

1. Ghana Statistical Service, Ghana Health Service, IFC Internnational. Ghana Demographic and Health Survey 2014. Accra, Ghana, 2015.

2. The American College of Obstetricians and Gynecologists. Frequently Asked questions: Long-Acting Reversible Contraception (LARC): IUD and Implant. The American College of Obstetricians and Gynecologists. 2016. https://www.acog.org/Patients/FAQs/LongActing-Reversible-Contraception-LARC-IUD-and-Implant. Accessed July 7, 2017.

3. Jacobstein R, Pile JM. Hormonal immplants: Service Delivery Considerations for an Improved and Increasingly Popular Method. 2010. http://www.respond-project.org/pages/files/6_pubs/technical_brief s/Technical-Brief-1-Hormonal-Implants-March2010-final-for-web. pdf. Accessed June 20, 2019.

4. Isley MM, Edelman A. Contraceptive implants: An overview and update. Obstet Gynecol Clin North Am. 2007;34:73-90.

5. Duvall S, Thurston S, Weinberger M, et al. Scaling up delivery of contraceptive implants in sub-Saharan Africa: Operational experiences of Marie Stopes International. Glob Health Sci Pract. 2014;2:72-92.

6. Elias B, Hailemariam T. Implants contraceptive utilization and factors associated among married women in the reproductive age group (18-49 year) in Southern Ethiopia. J Womens Health Care. 2015;4:281.

7. K4Health. Toolkits. The Knowledge for Health (K4Health) Project. https: //www.k4health.org/toolkits/implants/jadelle $\AA$-implants. Accessed October 20, 2017.

8. Charyeva Z, Oguntunde O, Orobaton N, et al. Task shifting provision of contraceptive implants to community health extension workers: Results of operations research in Northern Nigeria. Glob Health Sci Pract. 2015;3:382-394.
9. Chin-quee D, Bratt J, Malkin M, et al. Building on safety, feasibility, and acceptability: The impact and cost of community health worker provision of injectable contraception. Glob Health Sci Pract. 2013;1:316-327.

10. Kusi-appouh D, Acquah A, Tapsoba P. The Jagged Road to a Policy Change: Increasing Access to Family Planning using Community Health Nurses. In: UAPS 7th APC Submission. 2015:1-14.

11. Jardin A, Pham MT, Mallet A, et al. A medical simulator for subcutaneous contraceptive implant insertion. Med Eng Phys. 2008;30:1134-1142.

12. World Health Organization. WHO recommendations: Optimizing health worker roles to improve access to key maternal and newborn health interventions through task shifting. Geneva: WHO; 2012.

13. Tapsoba P, Aboagye PK. Increasing Access to Family Planning in Ghana through Policy Change: Task-Sharing to Enable Auxiliary Nurses to Provide Contraceptive Implant Services. Ghana, Accra: The Population Council, Inc.; 2014.

14. Perosky J, Richter R, Rybak O, et al. A low-cost simulator for learning to manage postpartum hemorrhage in Rural Africa. Simul Healthc. 2011;6:42-47.

15. Dieter GE, Schmidt LC. Engineering Design, 5th edn. New York, USA: McGraw-Hill; 2013:196-261.

16. MERCK. Nexplanon Etonogestrel Implant Implanon Medical Training Kit MERCK Program. https://www.ebay.com/itm/Nexplanon-Etono gestrel-Implant-Implanon-Medical-Training-Kit-MERCK-Progr am-/332222076203. Accessed May 3, 2018.

17. Lin $\mathrm{Y}, \mathrm{Wang} \mathrm{X}, \mathrm{Wu} \mathrm{F}$, et al. Development and validation of a surgical training simulator with haptic feedback for learning bone-sawing skill. J Biomed Inform. 2014;48:122-129.

18. Schubart JR, Erdahl L, Smith JS, et al. Use of breast simulators compared with standardized patients in teaching the clinical breast examination to medical students. J Surg Educ. 2012;69:416-422.

19. Khunger N, Kathuria S. Mastering surgical skills through simulation-based learning: Practice makes one perfect. J Cutan Aesthet Surg. 2016;9:27-31.

20. Lateef F. Simulation-based learning: Just like the real thing. J Emerg Trauma Shock. 2010;3:348-352.

21. Solymos O, O'Kelly P, Walshe CM. Pilot study comparing simulationbased and didactic lecture-based critical care teaching for final-year medical students. BMC Anesthesiol. 2015;15:153.

22. Dodge LE, Hacker MR, Averbach SH, et al. Assessment of a highfidelity mobile simulator for intrauterine contraception training in ambulatory reproductive health centres. J Eur CME. 2016;5:30416. 